\title{
Advanced roles in respiratory healthcare science: it's not just spirometry
}

Advanced clinical practitioner (ACP) roles in nursing began as early as the 1970s. Health Education England, along with National Health Service (NHS) Improvement and NHS England, have recently published a definition of and standards for multiprofessional advanced clinical practice [1]. They define these practitioners as "experienced, registered health and care practitioners...a level of practice characterised by a high degree of autonomy and complex decision making...underpinned by a masters level award or equivalent." These roles are now common place in healthcare professions such as nursing, physiotherapy and occupational therapy.

The document reshaping the workforce to deliver the care patients need [2] highlights how expanding the roles of the nonmedical workforce can help manage the growing burden of chronic disease more effectively and efficiently. Benefits of $A C P$ roles in nursing are said to include:

- improved patient access and experience

- addressing shortfalls in the medical workforce

- professional continuity

- career opportunities

- improved job satisfaction

However, there are issues with regards to the funding of the training required and the current lack of a regulatory or competency framework.

In 2010, the modernising scientific careers framework [3] was implemented to improve and streamline the training and education of the healthcare science workforce. It established the role of the healthcare scientist (HCS) at career framework level 6 and introduced the higher specialist scientific training programme leading to a higher specialist register and potential employment as a consultant clinical scientist.

As with other ACPs, clinical scientists complete a Master's degree (Clinical Science) or can register as a clinical scientist by demonstrating equivalence. The Academy for Healthcare Science [4] describes their roles as complex scientific and clinical roles, requiring highly skilled staff who can exercise clinical judgement about complex facts and clinical situations. Unlike nursing ACPs, clinical scientists are able to register with a statutory body, the Health and Care Professions Council.

Despite this, respiratory physiologists and scientists are often still regarded as "the ones that perform the FVC" or the "spirometry technician". We aim to highlight the current expanding roles of HCSs working in respiratory medicine, and how the skills and expertise developed through years of scientific training can be used to deliver a range of services within the primary and secondary care settings. This is particularly important considering the NHS interim People's Plan [5]. This plan describes how the future NHS workforce will be much more multidisciplinary, with individuals having fewer linear career pathways. The plan also recognises that the future NHS will rely on multiprofessional clinical teams rather than treating the workforce as separate groups of professions. Respiratory HCSs

@ERSpublications

Respiratory physiologists and clinical scientists are often still regarded as the "spirometry technician". @shakeyjs, @resp_edward, @Breath_to_win and @melaniebryce11 report on the evolving role of healthcare scientists working in respiratory physiology. http://bit.ly/2IQjlsc

Cite as: Shakespeare J, Parkes E, Bryce $M$, et al. Advanced roles in respiratory healthcare science: it's not just spirometry. Breathe 2019; 15: 267-269.

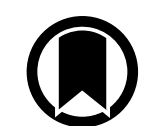

CrossMark

(c) ERS 2019 
can actively contribute to the management of patients in roles historically undertaken by others. However, this is not about bridging the gap in other professional groups; rather, this creates a larger workforce for delivery of patient care and at the same time, fulfils the need of the modern NHS workforce of being more flexible and adaptive. By valuing the healthcare science workforce, and recognising the skills and experience they have, and the advanced roles that they can undertake, we are more likely to recruit and to retain our current workforce.

HCSs are now equipped with specialist clinical skills that enable the assessment, diagnosis and treatment of patients. In addition, HCSs are now being upskilled to interpret full blood counts and chest radiographs, take detailed medical histories, and perform respiratory examinations, allowing complex clinical decisions to be made. This, of course, may be standard practice in some allied health professional domains but for HCSs working predominately within outpatient respiratory and sleep science services, this appreciably extends the scope of clinical practice.

\section{Advanced respiratory HCSs and primary care diagnostics}

Respiratory HCSs are leading primary care diagnostic services, moving traditional secondary care services closer to the patient [6]. These advanced practitioners are using their newly developed clinical assessment skills, in addition to their scientific training, to assess and diagnose patients in primary care. This provides general practitioners (GPs) with the information they require to manage their patients effectively, significantly reducing the requirement to refer to secondary care. Audits of the service at North West Anglia NHS Trust have demonstrated that $>70 \%$ of patients can be discharged back to the referring GP without the requirement for secondary care referral. 6-month follow-up demonstrated that $<1 \%$ of these patients subsequently went on to be referred to a chest clinic. This has resulted in significant cost savings and the generation of secondary care capacity.

\section{Advanced respiratory HCSs and acute noninvasive ventilation}

Advanced HCSs are now being recognised for clinically meaningful contributions towards the delivery of safe, high-quality and innovative acute ventilatory care. Indeed, some acute noninvasive ventilation (NIV) services are led by HCSs using highlevel leadership and clinical skills. Furthermore, HCSs are now a driving force of research, audit and innovation, which is demonstrated by the implementation of quality improvement initiatives such as the interdisciplinary acute NIV team at University Hospitals Coventry and Warwickshire (UHCW) NHS Trust, Coventry, UK. This team comprises HCSs, nurses, physiotherapists and physicians. It was developed in response to the acute NIV National Confidential Enquiry into Patient Outcome and Death [7] and the intention was to develop a culture of positive change and improve patients' outcomes. Recent audits using British Thoracic Society quality standards [8] have shown a reduction in inpatient mortality, an increase in treating the right patients at the right time, with HCSs initiating NIV in $>50 \%$ of patients. HCSs are almost certainly breaking professional boundaries, and are now showcasing their newly established clinical and nonclinical skills in healthcare settings other than outpatient departments, including accident and emergency and general critical care wards.

\section{Advanced respiratory HCSs and long-term ventilation}

Many respiratory physiology departments have been involved in long-term ventilation; however, historically, this involvement has been limited to downloading compliance data, blood gas analysis and titration of settings. In some centres, this role has developed considerably, with advanced HCSs becoming integral to the clinical assessment and monitoring of patients in the clinic environment [9]. A significant advantage of the close relationship with physiologists is the ability to provide patients with a "one-stop" NIV service with all required investigations occurring at the same appointment as their clinical assessment.

HCSs are now able to directly manage patients with long-term and palliative conditions such as motor neurone disease. They manage their therapy, and make important clinical decisions regarding starting and stopping ventilatory support. Their clinical development and experience enable them to provide advice and support outside of their tradition scope of practice. At UHCW NHS Trust, HCSs can, for example, have conversations with patients about the timing of percutaneous endoscopic gastrostomy and are able to provide ventilatory support for patients during these procedures.

We have highlighted the evolved role and improved scope of clinical practice of HCSs working in respiratory medicine. They now work across a wide range of clinical areas, including emergency and acute medicine, long-term ventilation, and primary care. This is far removed from the historical perceptions of a spirometry technician. Nationally, this is an underutilised workforce whose skills are not widely recognised. These examples have illustrated how HCSs are embracing the idea 
of an evolving NHS workforce that works across professional groups and not in professional silos.

Although we have showcased the advanced roles of HCSs, this should not detract from the clinically significant data that is obtained from the performance of quality assured diagnostic testing that includes pulmonary function testing and cardiopulmonary exercise testing, which are integral to the diagnosis and management of respiratory disease.

\section{Affiliations}

\section{Joanna Shakespeare ${ }^{1}$, Edward Parkes ${ }^{1}$, Melanie Bryce ${ }^{2}$, James Hull ${ }^{3}$}

${ }^{1}$ Dept of Respiratory and Sleep Sciences, University Hospitals Coventry and Warwickshire, Coventry, UK. ${ }^{2}$ Clinical Physiology Dept, North West Anglia NHS Foundation Trust, Huntingdon, UK. ${ }^{3}$ Dept of Respiratory Medicine, Royal Brompton and Harefield NHS Trust, London, UK.

\section{Conflict of interest}

None declared.

\section{References}

1. Health Education England. Multi-professional framework for England. www.hee.nhs.uk/printpdf/our-work/advancedclinical-practice/multi-professional-framework. Date last updated: 2019

2. Imison C, Castle-Clarke S, Watson R. Reshaping the workforce to deliver the care patients need. www.nuffieldtrust.org.uk/ research/reshaping-the-workforce-to-deliver-the-carepatients-need. Date last updated: 2016

3. Hill S, Taylor D, Hannigan B, et al. Modernising scientific careers: the UK way forward. https://assets.publishing. service.gov.uk/government/uploads/system/uploads/ attachment_data/file/138326/dh_113990.pdf. Date last updated: 2010

4. Academy for Healthcare Science. Career Framework for Healthcare Scientists. www.ahcs.ac.uk/about/the-healthcarescience-industry/career-framework-for-healthcarescientists/. Date last updated: 2017
5. NHS Improvement. Interim NHS People Plan. https:// improvement.nhs.uk/resources/interim-nhs-people-plan/. Date last updated: 2019

6. NHS. The NHS Long Term Plan. www.longtermplan.nhs. uk/wp-content/uploads/2019/01/nhs-long-term-planjune-2019.pdf. Date last updated: 2019

7. Juniper M, Ellis G, Smith NCE, et al. Inspiring change. A review of the quality of care provided to patients receiving acute non-invasive ventilation. www.ncepod.org.uk/2017report2/downloads/ InspiringChange_FullReport.pdf. Date last updated: 2017

8. Davies M, Allen M, Bentley A, et al. British Thoracic Society quality standards for acute non-invasive ventilation in adults. BMJ Open Respir Res 2018; 5: e000283.

9. Shakespeare J. The trials and tribulations of establishing a domiciliary NIV service. www.respiratoryfutures.org.uk/ features/the-trials-and-tribulations-of-establishing-adomiciliary-niv-service/. Date last updated: 2019 